\section{A simple technique for observing germ tube formation in Candida albicans}

\section{R. Y. CARTWRIGHT Public Health Laboratory, Guildford, Surrey}

Candida albicans is identified in most laboratories by the rapid formation of germ tubes when it is grown in human serum (Taschdjian et al, 1960).

Demonstration of the production of germ tubes on solid media, $1 \%$ bactopeptone in $2 \%$ agar (Joshi and Gavin, 1974), and the appearance of other yeasts on a variety of carbohydrate media (Joshi et al, 1975) has led to the development of a simple technique for observing germ tube production.

\section{Material and Methods}

Hyland Immunoplates (085-710) are washed in $70 \%$ Industrial Methylated Spirit (IMS) and dried. The plates are completely filled with approximately $4 \mathrm{ml}$ of $0.1 \%$ glucose in $2 \%$ New Zealand agar. Overfilling should be avoided as this prevents the lid being replaced. Prepared plates will keep for up to seven days at $+4^{\circ} \mathrm{C}$; they should be stored with the agar surface facing down.

The plate is inoculated by emulsifying part of a yeast colony which is less than 24 hours old, then

Received for publication 21 October 1975



Fig 1

Fig 1 A Hyland Immunoplate on a backing sheet used when testing yeasts for germ tube formation.

Fig 2 A strain of Candida albicans after three hours' incubation at $37^{\circ} \mathrm{C} . \times 600$

Fig 2

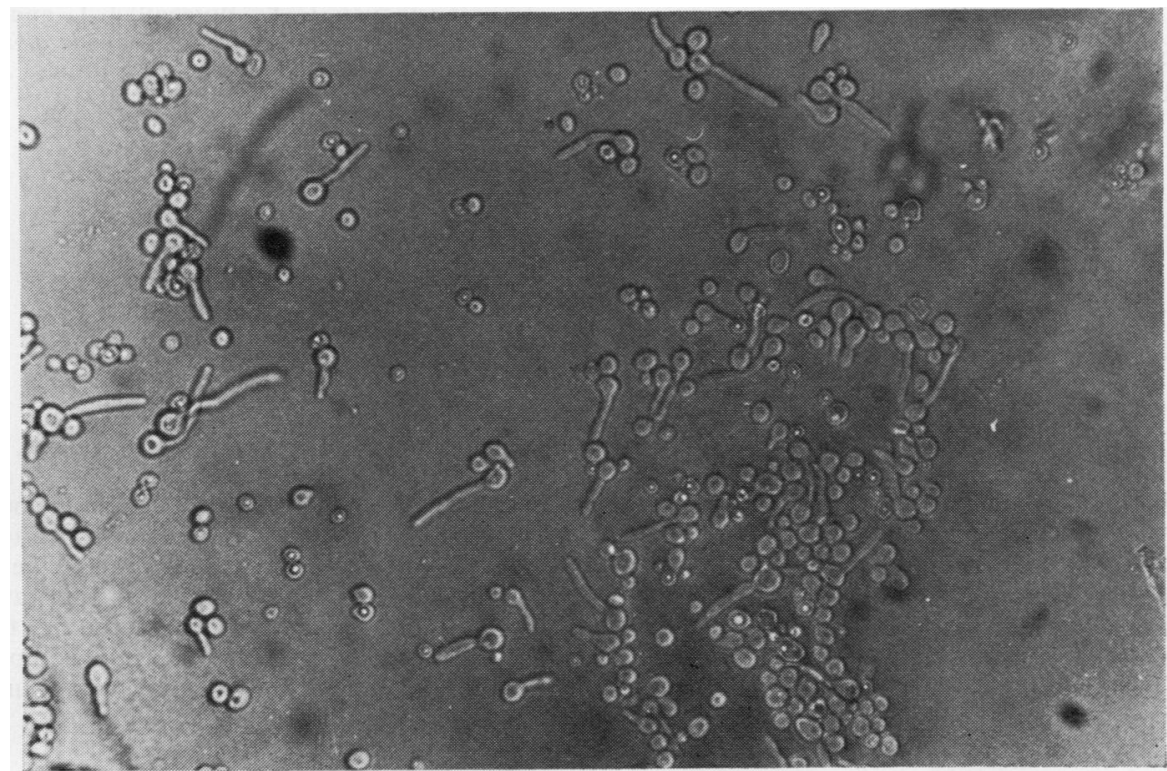


very lightly streaking it across the surface of the agar. Nine parallel streaks can easily be made on each plate - eight test strains and a positive control strain. A backing sheet is used for identification and to record the results (fig 1). Coverslips (22 $\mathrm{mm} \times$ $22 \mathrm{~mm}$ ) are cut in half, cleaned in $70 \%$ IMS, and sterilized in a hot air oven. False negative results were obtained when the coverslips were stored in the spirit and flamed just before use. The coverslips are placed over the streaks; three half coverslips fit each plate. Whole coverslips are not used as they are the width of the plate and can be difficult to position. The coverslip must be in contact with the agar surface.

The plate is placed on a microscope stage and the inocula are inspected. The yeast cells should be well separated from each other. The lid is replaced and the plate is incubated at $37^{\circ} \mathrm{C}$ for $2 \frac{1}{2}-3$ hours and then re-examined. The presence of germ tubes can be readily seen using a $\times 10$ or $\times 25$ objective (fig 2).

Germ tube negative strains should be studied for evidence of growth or cell division; further incubation for two hours may be necessary. Strains not showing evidence of growth should be subcultured and re-tested using a colony not more than 18 hours old.

The production of chlamydospores can be observed if the plates are kept at room temperature and examined daily for three days. The plates can be re-used by lifting out the agar, then washing them in $70 \%$ IMS.

\section{Results and Comments}

Two hundred and fifty-seven yeasts isolated in a routine clinical laboratory were examined; $96 \cdot 8 \%$
(249) produced germ tubes after three hours' incubation, and 15 of these strains were examined in the API Candida system. They all identified as $C$. albicans. The 18 germ tube negative strains were examined in the API Candida system; 11 were Torulopsis glabrata, four Candida krusei, two Candida tropicalis, and one has not yet been identified.

This technique provides a convenient and reliable method for assessing germ tube production in yeasts. The use of serum and other liquid media requires incubation in tubes, then the transfer of a drop onto a microscope slide for examination. The introduction of solid media enables the growing yeasts to be observed directly. The manipulation of petri dishes on a microscope stage is not, however, easy, and objectives can easily be pushed into the medium. The use of an immunodiffusion plate overcomes this difficulty, and a single field can be repeatedly examined. Oil immersion objectives can be used to follow the growth of individual yeast cells.

The test should always be carried out using a colony 18-24 hours old. False negative results may be obtained if older colonies are used. The positive control strain must be subcultured daily.

\section{References}

Joshi, K. R., Bremner, D. A., Parr, D. N., and Gavin, J. B. (1975). The morphological identification of pathogenic yeasts using carbohydrate media. J. clin. Path., 28, 18-24.

Joshi, K. R. and Gavin, J. B. (1974). A simple laboratory method for the rapid identification of Candida albicans. Pathology, 6, 231-233.

Taschdjian, C. L., Burchall, J. J., and Kozinn, P. J. (1960). Rapid identification of Candida albicans by filamentation on serum and serum substitutes. Amer. J. Dis. Child., 99, 212-215.

\section{Book reviews}

Biochemistry of Human Cancer. By Oscar Bodansky. (Pp. xiv +657 ; £18.95.) London: Academic Press. 1975.

A flood of biochemical detail related to human cancer continues to pour out of research laboratories the world over. This book sets out to describe and evaluate these data in relation to different types of human cancer.

Introductory chapters describe general metabolic, enzymic, and immunochemical characteristics. The remainder of the book is arranged according to the organ or anatomical site of the neoplasms discussed. The biochemistry of brain tumours is not included. The review under each heading is concise but not superficial and selects from work published up to and including 1973.

The main value of the book is in the clinical field and where, as for example in breast cancer, a common disease is asईciated with a plethora of sometimes conflicting biochemical data, it would ha been helpful to have had a more incisige analysis of the value of laboratory investigation in patient management. For $\bar{\sigma}^{-a}$ single author to have covered such a wide field so competently, however, is sonfithing of a tour-de-force.

C. B. CAMER@i 